\title{
ON ZEROS OF BOUNDED DEGREE OF SYSTEMS OF HOMOGENEOUS POLYNOMIAL EQUATIONS
}

\author{
Georg Eulering and Martin Krüskemper
}

Let $F$ be a finite or algebraically closed field and $R=$ $F\left[T_{1}, \ldots, T_{s}\right]$, the polynomial ring in $T_{1}, \ldots, T_{s}$ over $F$. Then by Tsen-Lang, any system of homogeneous polynomials $f_{1}(X), \ldots$, $f_{r}(X) \in R[X]$ of degree $d$, where $X=\left(X_{1}, \ldots, X_{n}\right)$, has a nontrivial common zero in $R^{n}$ provided the number of variables $n$ is sufficiently large. In this note we want to give an effective bound $B$ such that there exists a zero $0 \neq\left(a_{1}, \ldots, a_{n}\right) \in R^{n}$ with $\max \left\{\operatorname{deg}\left(a_{1}\right), \ldots, \operatorname{deg}\left(a_{n}\right)\right\} \leq B$. The bound depends on $d, r, s$ and the maximal degree of the coefficients of the $f_{j}$ where $j=1, \ldots, r$. In particular, if $F$ is finite, a common zero can be computed effectively.

Let $F$ be an arbitrary field. We fix $d, n, r, s \in \mathbb{N}$. Let $R_{s}=F\left[T_{1}, \ldots, T_{s}\right]$ be the polynomial ring in $T_{1}, \ldots, T_{s}$ over $F$. Recall that if $f \in R_{s}$ is a

polynomial of total degree $d$ then it has $\left(\begin{array}{c}s+d \\ s\end{array}\right)$ coefficients. A homogeneous $f \in R_{s}$ of degree $d$ has $\left(\begin{array}{c}s+d-1 \\ s-1\end{array}\right)$ coefficients. If $\alpha=\left(a_{1}, \ldots, a_{n}\right) \in R_{s}^{n}$, we define the degree of $\alpha, \operatorname{deg}(\alpha):=\max \left\{\operatorname{deg}\left(a_{1}\right), \ldots, \operatorname{deg}\left(a_{n}\right)\right\}$ where $\operatorname{deg}\left(a_{j}\right)$ denotes the total degree of $a_{j}=a_{j}\left(T_{1}, \ldots, T_{s}\right)$ for $j=1, \ldots, r$.

Let $X=\left(X_{1}, \ldots, X_{n}\right), R_{s}[X]=R_{s}\left[X_{1}, \ldots, X_{n}\right]$ where $X_{j}$ are new variables. If $f_{1}(X), \ldots, f_{r}(X) \in R_{s}[X]$ are forms (i.e., homogeneous polynomials) of degree $d$, then a non-trivial common zero of $f_{1}, \ldots, f_{r}$ is some $0 \neq \alpha=\left(a_{1}, \ldots, a_{n}\right) \in R_{s}^{n}$ such that $f_{j}\left(a_{1}, \ldots, a_{n}\right)=0$ for any $j=1, \ldots, r$. Let $\operatorname{deg}\left(f_{1}, \ldots, f_{r}\right)$ denote the maximal degree of the coefficients of the $f_{j}$ where $j=1, \ldots, r$.

Let $i \geq 0$. A field $F$ is called $C_{i}$-field if any form $f \in F[X]=F\left[X_{1}, \ldots, X_{n}\right]$ of degree $d$ has a non-trivial zero provided $n>d^{i}$. See $[\mathbf{L}]$. Recall that $F$ is $C_{0}$ if and only if $F$ is algebraically closed. Further, if $F$ is $C_{i}$ then $F\left(T_{1}, \ldots, T_{s}\right)$ is $C_{i+s}$. A survey on Tsen-Lang theory can be found in [Lo] for instance. In $[\mathbf{P} 1]$, Pfister defined the property $C_{i}(d)$ for any $d \in \mathbb{N}$ : A field $F$ satisfies $C_{i}(d)$ if any system of $r$ forms of degree $d$ over $F$ in $n$ variables has a non-trivial common zero in $F$ provided $n>d^{i} r$. For instance the field of real numbers $\mathbb{R}$ is not $C_{i}$ for any $i$ but $\mathbb{R}$ is $C_{0}(d)$ for all odd $d$. See $[\mathbf{P} 1]$ for a proof.

Let $F$ be $C_{i}$. We ask the following question: Given $f_{1}, \ldots, f_{r} \in R_{s}[X]$ of degree $d$, is there a bound $B$ depending on $i, d, r, s$ and $\operatorname{deg}\left(f_{1}, \ldots, f_{r}\right)$ 
such that there exists a common non-trivial zero $\alpha \in R_{s}^{n}$ with $\operatorname{deg}(\alpha) \leq B$ ? Following Cassels [C], Prestel showed that for any field $F$, a quadratic form $f \in F\left[T_{1}\right][X]$ which has a non-trivial zero, has a zero of degree $\leq \operatorname{deg}(f)(n-$ $1) / 2$. This bound is best possible. If the polynomial ring is in two or more variables, then $[\mathbf{P}]$, Theorem 2 shows that there is no bound anymore for arbitrary base field $F$. In connection with problems of real algebraic geometry, Mahé $([\mathrm{M}])$ and the first author both needed to find zeros of bounded degree of a quadratic form with values in $C\left[T_{1}, \ldots, T_{s}\right]$ where $C$ is algebraically closed. In the following we want to give such a bound $B$ for arbitrary systems of forms by a modification of Lang's arguments in [L]: We will replace Lang's induction on $s$ by some effective computations.

For some important remarks the authors would like to thank L. Bröcker and A. Pfister.

Let $a \in \mathbb{R}$. Let $n \in \mathbb{Z}$ such that $n-1<a \leq n$. Then we set $\lceil a\rceil:=n$.

Proposition 1. Suppose $F$ is $C_{i}(d)$. Let $R_{s}=F\left[T_{1}, \ldots, T_{s}\right]$ and $f_{1}(X), \ldots$, $f_{r}(X) \in R_{s}[X]$ be forms of degree $d$. Let $g:=\operatorname{deg}\left(f_{1}(X), \ldots, f_{r}(X)\right)$. If $n>r d^{i+s}$, then the system $f_{1}, \ldots, f_{r}$ has a non-trivial common zero $\alpha \in R_{s}^{n}$ such that $\operatorname{deg}(\alpha) \leq\lceil g / \tau\rceil$ where $\tau:=\sqrt[s]{n /\left(d^{i} r\right)}-d$.

Proof. For any $\beta=\left(l_{1}, \ldots, l_{s}\right)$ where $0 \leq l_{j}$, we set $T^{\beta}:=T_{1}^{l_{1}} \ldots T_{s}^{l_{s}}$. For $m \in \mathbb{N}$ we set $\Lambda(m):=\left\{\beta=\left(l_{1}, \ldots, l_{s}\right) \mid 0 \leq l_{j}\right.$ for $j=1, \ldots, s, \sum_{j=1}^{s} l_{j} \leq$ $m\}$. Now we fix some $m$. For any $\beta \in \Lambda(m)$ we choose $n$ new variables $X_{1, \beta}, \ldots, X_{n, \beta}$. We substitute $X_{j}=\sum_{\beta \in \Lambda(m)} X_{j, \beta} T^{\beta}$ for $j=1, \ldots, n$ in $f_{1}, \ldots, f_{r}$ and obtain for $k=1, \ldots r$,

$$
f_{k}\left(\left\{X_{j, \beta}\right\}\right)=\sum_{\beta \in \Lambda(d m+g)} f_{k, \beta} T^{\beta}
$$

where the $f_{k, \beta}$ are homogeneous over $F$ of degree $d$ in $n\left(\begin{array}{c}s+m \\ s\end{array}\right)$ variables $X_{j, \beta}$. The number of forms $f_{k, \beta}$ is $r\left(\begin{array}{c}d m+g+s \\ s\end{array}\right)$. Now we set $m:=\lceil g / \tau\rceil$ where $\tau:=\sqrt[s]{n /\left(d^{i} r\right)}-d$. We claim

$$
\text { (*) } n\left(\begin{array}{c}
s+m \\
s
\end{array}\right)>d^{i} r\left(\begin{array}{c}
d m+g+s \\
s
\end{array}\right) \text {. }
$$

Then the system of forms $f_{k, \beta}$ has a non-trivial common zero in $F^{n\left(\begin{array}{c}s+m \\ s\end{array}\right)}$ and a substitution proves the first statement of Proposition 1. To show $(*)$, note 
that $g \leq m \tau$ and hence

$$
\begin{aligned}
s ! d^{i} r\left(\begin{array}{c}
d m+g+s \\
s
\end{array}\right) & =d^{i} r \prod_{j=1}^{s}(d m+g+j) \\
& \leq d^{i} r \prod_{j=1}^{s}((d+\tau) m+j) \\
& <d^{i} r \prod_{j=1}^{s}(d+\tau)(m+j)=n \prod_{j=1}^{s}(m+j)=s ! n\left(\begin{array}{c}
s+m \\
s
\end{array}\right) .
\end{aligned}
$$

Since any field $F$ is $C_{0}(1)$ we obtain:

Corollary 1. Let $F$ be a field and $R_{s}=F\left[T_{1}, \ldots, T_{s}\right]$. Let $f_{1}(X), \ldots$, $f_{r}(X) \in R_{s}[X]$ be linear forms. Let $g:=\operatorname{deg}\left(f_{1}(X), \ldots, f_{r}(X)\right)$. If $n>r$, then the system $f_{1}, \ldots, f_{r}$ has a non-trivial common zero $\alpha \in R_{s}^{n}$ such that $\operatorname{deg}(\alpha) \leq\lceil g / \tau\rceil$ where $\tau:=\sqrt[s]{n / r}-1$. The last bound is best possible if $s=1$ and $n=r+1$.

Proof. If $s=1$ and $n=r+1$, then the above bound is best possible: Consider the system of $r$ linear forms $X_{1}-T_{1}^{g} X_{2}, X_{2}-T_{1}^{g} X_{3}, \ldots, X_{r}-T_{1}^{g} X_{r+1}$.

\section{Remarks.}

(1) If $F$ is a finite field then $F$ is $C_{1}$. If $n>r d^{s+1}$, then Proposition 1 shows that a non-trivial common zero of the system $f_{1}, \ldots, f_{r}$ can be computed effectively, since only finitely many $\alpha \in R_{s}^{n}$ have to be checked.

(2) Prestel's counter example is defined over the field $\mathbb{R}$ of real numbers which does not satisfy any property $C_{i}$. If the base field $F$ is arbitrary one should expect that unless $d=1$ or $r=s=1, d=2$ there exist no bounds on the size of a zero of a system $f_{1}, \ldots, f_{r}$. The case $r=s=$ $1, n=2$ is one more exception:

(3) Let $s=1$ and $f_{1}\left(X_{1}, X_{2}\right) \in R_{1}\left[X_{1}, X_{2}\right]$ be homogeneous of degree $d$. We show that there exists a non-trivial zero of $f_{1}$ of degree $\leq$ $g=\operatorname{deg}\left(f_{1}\right)$ provided $f_{1}$ has a non-trivial zero. Wlog we may assume $(a / b, 1)$ is the zero of $f_{1}$ where $a, b \in R, b \neq 0$. Then Gauß' theorem shows that $f_{1}\left(X_{1}, 1\right)=h\left(X_{1}\right)\left(X_{1}-c\right)$ where $h \in R_{1}\left[X_{1}\right]$ and $c \in$ $R_{1}, \operatorname{deg}(c) \leq g$. Hence $(c, 1)$ is a zero of $f_{1}\left(X_{1}, X_{2}\right)$. Of course the bound is best possible.

If the base field $F$ is finite, we can apply a stronger version of the theorem of Chevalley, Warning. If we apply [LN], Theorem 6.11, page 274 we can improve the statement of Proposition 1 as follows: 
Corollary 2. Let $F$ be a finite field with $q$ elements. Let $R_{s}=F\left[T_{1}, \ldots, T_{s}\right]$. Let $f_{1}(X), \ldots, f_{r}(X) \in R_{s}[X]$ be forms of degree d. Let $g:=\operatorname{deg}\left(f_{1}(X), \ldots\right.$, $\left.f_{r}(X)\right)$. Suppose $n>r d^{s+1}$ and $m \geq\lceil g / \tau\rceil$, where $\tau:=\sqrt[s]{n /(d r)}-d$. Then the system $f_{1}, \ldots, f_{r}$ has at least $q^{n\left(\begin{array}{c}s+m \\ s\end{array}\right)-r d(\underset{s}{d m+g+s})}$ different common zeros $\alpha \in R_{s}^{n}$ such that $\operatorname{deg}(\alpha) \leq m$. In particular if $s=1, n>r d^{2}, m \geq$ grd, we have $q^{\left(n-r d^{2}\right) m+n-r d(g+1)}$ different zeros of degree $\leq m$.

Example. Let $F$ be a finite field with $q$ elements. Proposition 1 shows that a quadratic form $f \in F\left[T_{1}\right][X]$ in $n \geq 5$ variables with $g:=\operatorname{deg}(f)$ has a non-trivial zero of degree $\leq 2 g$. Hence in this case, Prestel's bound gives the same result. By Corollary 2, if $m \geq 2 g$, there exist at least $q^{(n-4) m+n-2(g+1)}$ different zeros in $F\left[T_{1}\right]^{n}$ of degree $\leq m$.

By Tsen, if $F$ is $C_{i}$ and $F$ admits normic forms of level $i$ of arbitrary degree, then any homogeneous system $f_{1}(X), \ldots, f_{r}(X) \in F[X]$ of degree $d_{1}, \ldots, d_{r}$ has a non-trivial common zero provided $n>\sum_{j=1}^{r} d_{j}^{i}$. (See [Lo], page 158.) This holds for instance, if $F$ is finite for $i=1$.

Proposition 2. Let $F$ be a $C_{i}$ field such that $F$ admits normic forms of level $i$ of arbitrary degree. Let $R_{s}=F\left[T_{1}, \ldots, T_{s}\right]$. Let $f_{1}(X), \ldots, f_{r}(X) \in$ $R_{s}[X]$ be forms of degree $d_{1}, \ldots, d_{r}$. Let $g:=\operatorname{deg}\left(f_{1}(X), \ldots, f_{r}(X)\right)$. If $n>\sum_{j=1}^{r} d_{j}^{i+s}$, then the system $f_{1}, \ldots, f_{r}$ has a non-trivial common zero $\alpha \in R_{s}^{n}$ such that $\operatorname{deg}(\alpha) \leq\left\lceil s(g+2)^{s} n / \tau\right\rceil$ where $\tau:=n-\sum_{j=1}^{r} d_{j}^{i+s}$. In particular, $\operatorname{deg}(\alpha) \leq n s(g+2)^{s}$.

Proof. For $m \in \mathbb{N}$ we set $\Omega(m):=\left\{\beta=\left(l_{1}, \ldots, l_{s}\right) \mid 0 \leq l_{j} \leq m\right.$ for $j=$ $1, \ldots, s\}$. Now we fix some $m$. For any $\beta \in \Omega(m)$ we choose $n$ new variables $X_{1, \beta}, \ldots, X_{n, \beta}$. We substitute $X_{j}=\sum_{\beta \in \Omega(m)} X_{j, \beta} T^{\beta}$ for $j=1, \ldots, n$ in $f_{1}, \ldots, f_{r}$ and obtain for $k=1, \ldots r$,

$$
f_{k}\left(\left\{X_{j, \beta}\right\}\right)=\sum_{\beta \in \Omega(d m+g)} f_{k, \beta} T^{\beta}
$$

where the $f_{k, \beta}$ are homogeneous over $F$ of degree $d$ in $n(m+1)^{s}$ variables $X_{j, \beta}$. The number of forms $f_{k, \beta}$ is $\sum_{j=1}^{r}\left(d_{j} m+g+1\right)^{s}$. The system of forms $f_{k, \beta}$ has a non-trivial common zero if $n(m+1)^{s}>\sum_{j=1}^{r} d_{j}^{i}\left(d_{j} m+g+1\right)^{s}$, that is

$$
\sum_{l=0}^{s}\left(\begin{array}{l}
s \\
l
\end{array}\right) m^{l}\left(n-\sum_{j=1}^{r} d_{j}^{i+l}(g+1)^{s-l}\right)>0 .
$$

The highest coefficient of this polynomial in $m$ is $\tau>0$. Hence $(*)$ is equivalent to $\tau m^{s}>\sum_{l=0}^{s-1}-\left(\begin{array}{l}s \\ l\end{array}\right) m^{l}\left(n-\sum_{j=1}^{r} d_{j}^{i+l}(g+1)^{s-l}\right)$. Assume we have 


$$
\tau m>\sum_{l=0}^{s-1}\left|\left(\begin{array}{l}
s \\
l
\end{array}\right)\left(n-\sum_{j=1}^{r} d_{j}^{i+l}(g+1)^{s-l}\right)\right| .
$$

Then $(*)$ holds since we get

$$
\tau m^{s}>\sum_{l=0}^{s-1}\left(\begin{array}{l}
s \\
l
\end{array}\right) m^{l}\left|\left(n-\sum_{j=1}^{r} d_{j}^{i+l}(g+1)^{s-l}\right)\right| .
$$

We have $\left|n-\sum_{j=1}^{r} d_{j}^{i+l}(g+1)^{s-l}\right|<n(g+1)^{s-l}$. Thus (**) holds if

$$
\tau m \geq \sum_{l=0}^{s} n\left(\begin{array}{l}
s \\
l
\end{array}\right)(g+1)^{s-l}=n(g+2)^{s} .
$$

Now set $m:=\left\lceil(g+2)^{s} n / \tau\right\rceil$ and a substitution proves Proposition 2 .

\section{Remarks.}

(a) The last proof works also if $d_{1}=\cdots=d_{r}$ and $F$ is $C_{i}\left(d_{1}\right)$ or if all $d_{j}$ are odd and $F=\mathbb{R}$.

(b) Instead of forms and we could also consider polynomials without constant term if we replace $C_{i}$ by 'strongly $C_{i}$ '. See $[\mathbf{L}]$.

\section{References}

[C] J.W.S. Cassels, Bounds for the least solution of homogeneous quadratic equations, Proc. Camb. Phil. Soc., 51 (1995), 262-264.

[L] S. Lang, On quasialgebraic closure, Ann. of Math., 55 (1952), 373-390.

[LN] R. Lidl and H. Niederreiter, Finite fields, Addison-Wesley, London, Amsterdam, Don Mills, Sydney, Tokio, 1983.

[Lo] F. Lorenz, Einführung in die Algebra, Teil 2, BI, Mannheim, Wien, Zürich, 1990.

[M] L. Mahé, unpublished preprint.

[P1] A. Pfister, Systems of quadratic forms, Bull. Soc. Math. France Memoire, 59 (1979), 115-123.

[P] A. Prestel, On the size of zeros of quadratic forms over rational function fields, J. Reine Angew. Math., 378 (1987), 101-112.

Received April 1, 1996.

MATHEMATISChes INSTITUT DER UNIVERSitÄT

EINSTEINSTRASSE 62

D-48149 Münster, Germany

E-mail address: kruskem@math.uni-muenster.de 